\title{
Mechanical, electrochemical and structural characteristics of friction stir spot welds of aluminium alloy 6063
}

\author{
Delphine Mulaba-Kapinga ${ }^{1}$, Kasongo Didier Nyembwe ${ }^{1}$, Omolayo Michael Ikumapayi ${ }^{2}{ }^{*}$ \\ and Esther Titilayo Akinlabi ${ }^{3}$ \\ ${ }^{1}$ School of Mining and Metallurgy and Mechanical Engineering, University of Johannesburg, Doornfontein Campus and \\ Auckland Park Campus, Johannesburg, South Africa \\ 2 Department of Mechanical Engineering Science, University of Johannesburg, Auckland Park Kingsway Campus, \\ Johannesburg,2006, South Africa \\ ${ }^{3}$ Pan African University for Life and Earth Sciences Institute (PAULESI), Ibadan, Nigeria
}

Received: 13 April 2020 / Accepted: 21 July 2020

\begin{abstract}
The work presents the friction stir spot welding (FSSW) of AA6063. The evolving properties due to the influence of process parameters and the efficacy of metallurgical, structural, mechanical, and electrochemical integrities were studied. FSSW was conducted on $2 \mathrm{~mm}$ thickness by varying the rotational speed of 600,900 and $1200 \mathrm{rpm}$ and the dwell time at 10 and $15 \mathrm{~s}$. The evolving microstructures, hardness, corrosion, shear tensile behaviours and X-ray diffraction characteristics of the as-received material and the welds were studied. As the tool rotational speed increased at a constant dwell time, a smooth and debris free spot welds were noticed, more HAZ formations became visible and more intermetallic phases of aluminium magnesium (AlMg) were formed although with very low peaks during structural assessment. Furthermore, the hardness values increased up to a certain limit and then decreased, the corrosion properties in artificial seawater (ASW) shown significant improvement on the spot-welded samples and the tensile shear strength was also improved. It would be recommended that spot welds at $900 \mathrm{rpm}$ and 10 and/or $15 \mathrm{~s}$ for applications where the hardness is significant imperative and at $1200 \mathrm{rpm}$ with 10 and/or $15 \mathrm{~s}$ dwell time where higher tensile shear strength is required and lastly, $1200 \mathrm{rpm}$ at $15 \mathrm{~s}$ where corrosion application is significant.
\end{abstract}

Keywords: Aluminium alloy / artificial seawater / friction stir spot welding / intermetallic phases / tensile shear strength

\section{Introduction}

Friction Stir Spot Welding (FSSW) as a linear modification of the FSW process has gained popularity to the extent of replacing the conventional Resistance Spot Welding (RSW) [1]. This practice produces a single spot welded joint from the overlapping adjacent material components $[2,3]$.

FSSW was built during the development of the Mazda RX-8 hood and rear door panel in 2003 and is a replacement method for spot welding of lightweight alloys. This welding method can weld aluminium alloy in a lap and butt position [4]. Thanks to its high product quality the FSSW is successfully applied in the automotive industry $[5,6]$. FSSW is used to weld soft, thin metals, such as aluminium,

\footnotetext{
* e-mail: Ikumapayi.omolayo@gmail.com
}

copper and magnesium alloy as well as different material combination, particularly those with a close melting point. FSSW has recently been extended to welding of materials which include steel, titanium, and nickel alloys; materials that have high melting points [7].

The FSSW process is made up of a tool shoulder and a cylindrical tool pin which is pressed against the two sheets which thus produced a weld. The tool shoulder is responsible for the heat which is generated during the FSSW process; the tool shoulder also inhibits the material removal. The shoulder size and its depression are important aspects during FSSW. The pin produces frictional heat, stirs the heated material and generates a deformation of the material around it. While the pin turns around it, it enters the plates, produces a plastic deformation while the plate moves on the line. The experimental set-up is depicted in Figure 1a while a joint called a weld is produced due to the friction between the tool and the sheets as represented in Figure 1b [8]. 


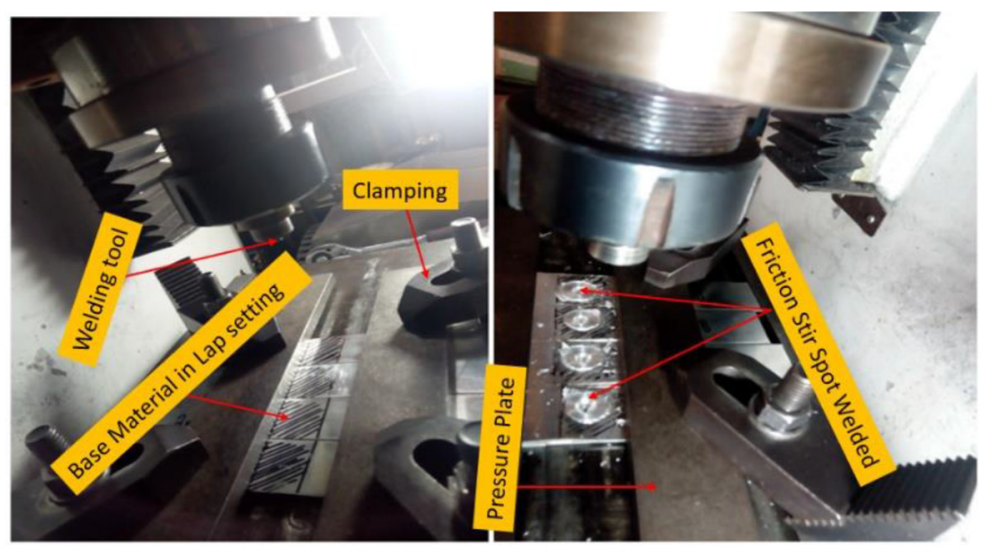

(a)

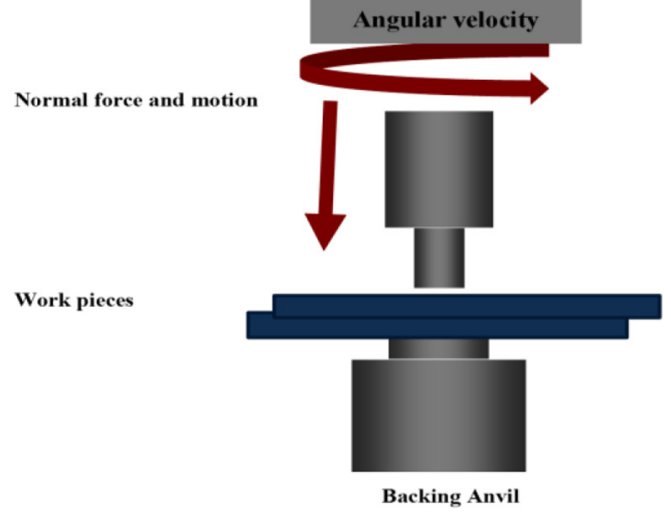

(b)

Fig. 1. (a) Experimental Setup of the FSSW operation (b) A schematic representation of the FSSW process.

Yuang [9] and Siddharth et al. [10] suggested that FSSW has found to be superior to the conventional welding techniques used to weld aluminium self-Piercing Riveting (SPR)and Resistance Spot Welding (RSW). This welding process can weld similar and dissimilar alloys and is more cost and energy effective and an alternate process compared to electrical resistant spot welding (ERSW). FSSW is better than RSW, MIG and mechanical joining process. FSSW has lower operational costs due to improved energy efficiency (a low heat input) and a virtual lack in consumables. A low distortion on the weld is produced and no porosity is formed on the weld during this process. FSSW equipment needs less surrounding infrastructure and no water, compressed air, toxic fumes or gases are exerted into the environment. Solidification cracking is prevented with this welding process, excellent bonding and a fine microstructure are produced, and no electrical transforming is needed. An additional attribute to this process is that it is an automatic and a high repeatability process [11].

\subsection{Effect of different FSSW process parameters}

According to Kulekci et al. [12], It is important to recognize how the FSSW parameters affect the welding integrity and how the joint weld works, because it is based on minimal literature. The mechanical properties are critical for FSSW joints and are mainly affected by the tool geometry and the process parameter. Welding parameters (see Fig. 2a) which have a strong impact on the joint strength during FSSW include pin profile, tool rotation rate, dwell time, plunge rate, shoulder depth and axial force [13]. Various distinctions are produced by the tool penetration and the dwell time; the heat generated during the FSSW process, how the material plastically deforms around the pin and the weld geometry; which evidently has an influence on the mechanical properties of the spot welds [14]. Welds produced at low spindle rates have fine mechanical properties compared to that at high spindle rates. The properties of the material improve as the rotational speed and the plunge rate is increased up to a certain level, and then it decreases. There is a positive relationship between the axial load and tensile strength. If the axial load increases then shear strength increases. With an increase in the dwell time, the joint strength increases up to a certain level, then it decreases [15]. Kumar et al. [16] documented a review on the optimization technique of FSW indicating that the important mechanical properties tested with FSW are the yield strength, UTS and the percentage elongation. Regarding the UTS, as the tool rotational speed, the axial force and then plunge rate decreases, the UTS of the weld joint decreases. Concerning the yield strength, as the defects in the weld decreases the tool rotational speed increase with a decrease in the plunge rate and a higher axial force. About the percentage elongation, as the defects in the weld zone of the joint increases the tool rotational speed increases decrease in the plunge rate and a higher axial force. The tool rotation rate is one of the most important parameters which play a major role in FSW, as the rotational tool rate, yield strength and percentage elongation increases, the UTS will also increase when the axial force increases and when the rotational tool rate increases a conical pin has the lowest tensile strength.

\subsection{FSSW of similar alloys}

Paidar et al. [6] investigated how varying the shoulder plunge depth and the rotational speed influenced the mechanical properties and failure modes of FSSW of aluminium sheets when FSSW was conducted on AA2024T3. It was found that the SZ had equiaxed fine grains which are produced by recrystallization that occurred where the tool pin rotated. The SZ defines the joint strength, how strong is the weld; hence the shoulder plunge depth and the rotational speed considerably affected the SZ. The shoulder plunge depth and the tool rotational speed increased maximum failure load; however, the influence of the rotational speed is far less than the shoulder plunge depth. Guler et al. [3] investigated how the tool geometry and the welding process parameters affect the static strength and hardness of FSSW AA 5754-H11 sheets. The pin profile, dwell time and the tool rotation rate were varied. Using the tapered pin, the tensile shear load increased together with the dwell time. The welds produced with the circular pin 

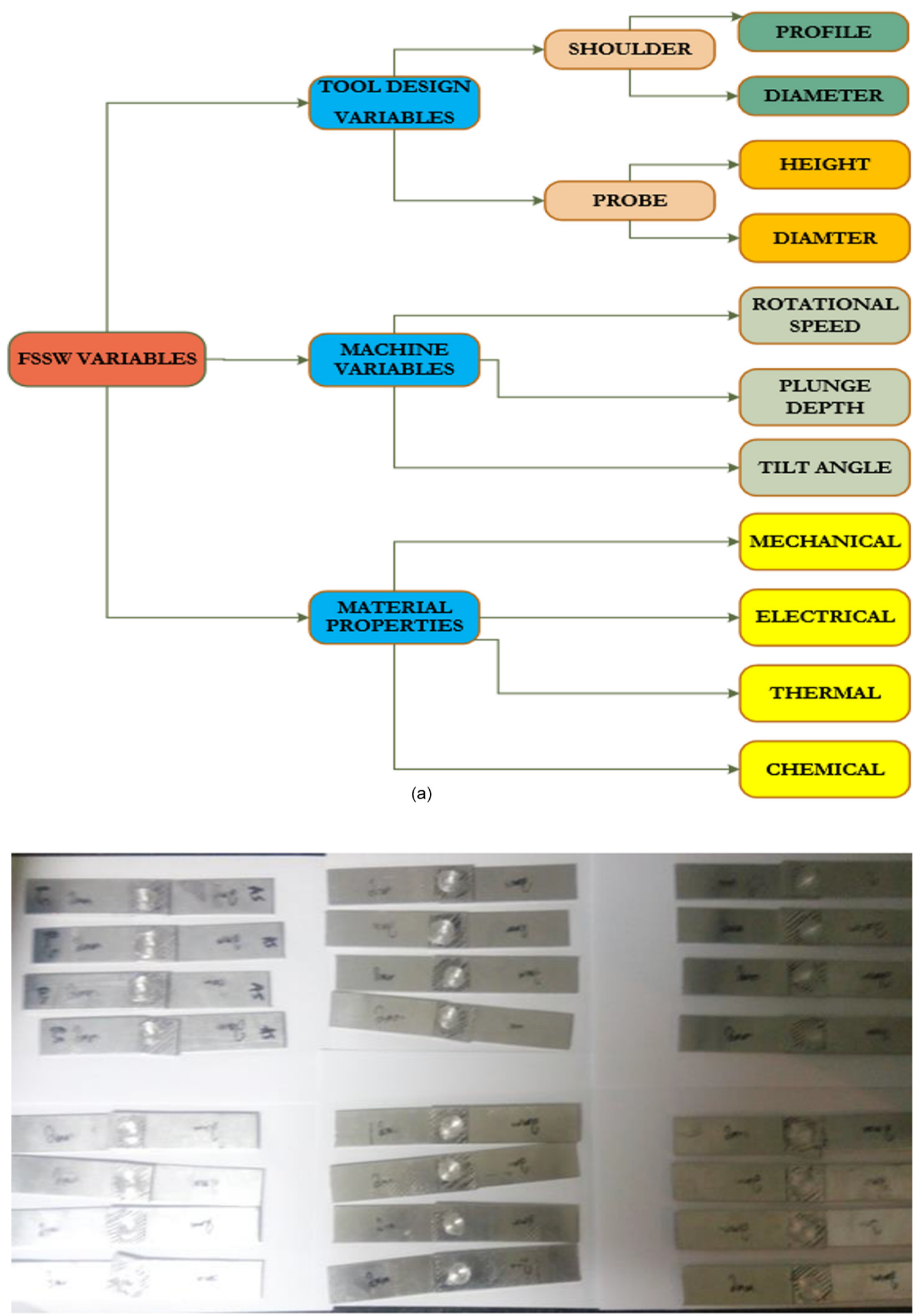

(b)

Fig. 2. (a) Schematic diagram of the variables used during FSSW and (b) Spot welds of samples prepared by FSSW. 
produced a higher tensile load because of better stirring on welding. There was no noticeable effect between the tool geometry and hardness.

Kulekci et al. [12] examined how the pin height, tool rotation rate and the dwell time effects on the tensile shear strength of FSSW of AA5005. The authors determined that the varied process parameters affected the shear strength of the alloy. As the tool pin height increased the shear strength increased. As the rotational tool rate increased the shear strength increased to a certain point and as the dwell time increased the shear strength decreased. Liu et al. [13] published a study on FSSW of AA2A12-T4, examining its microstructures and properties. In the study, the authors varying the tool rotational speed, plunge rate and shoulder plunge rate. It was revealed that the SZ, TMAZ and the HAZ were found in the joint. The lowest hardness was found in the TMAZ and the average hardness was found in the SZ which could be improved by increasing the welding heat input. As the rotational speed increased so did the shear load, with an increase in the plunge rate and with a decrease in the shoulder plunge depth, results showed that the tensile shear load decreased.

Tashkandi et al. [5] conducted spot welding of AA6061 by FSSW. The tool rotational speed, dwell time, shoulder diameter and pin length were varied. It was found that the lap shear fracture load (LSFL) increased as the rotational tool rate increased. With an increase in the dwell time, the LSFL decreased and the nugget pull-out fracture mode occurred at relatively higher LSFL. Hamzah et al. [17] investigated the effect of the pin shape and rotation rate on the behaviour and microstructure of the FSSW of AA6061. The pin profile and the tool rotation rate were varied. They concluded that the triangular pin showed the highest tensile shear testing. Testing displayed that with an increase in the rotational tool rate the shear load increased, however with it increasing more the shear load decreased, to some extent, for a specific pin shape. Hardness decreased gradually from the NZ through to the TMAZ and the HAZ caused by the fictional heat and the material deforming.

Buffa et al. [18] and Fratini et al. [19] focused on how different parameters of FSW affected AA 6082-T6 where the tool rotation rate and the plunge rate were varied. It was found that the FSW resulted in the recrystallized zone, HAZ and TMAZ. The tensile strength had a direct relationship with the plunge rate and the strength of the spot joint was less than that of the parent metal. The hardness reduced in the welded region where the softest areas were the HAZ, and the HAZ correspond to a failure location in the tensile test. Tunnel defects were detected where the weld nugget and the TMAZ were joined due to the high tool rotation rate and plunge rate, by optimizing such parameters the defects were avoided.

Chen et al. [20] attempted establishing a relationship between microstructural analysis, hardness, and corrosion sensitivity of FSSW AA6061. The FSSW process parameters were constant throughout creating the weld. The grain size observed reduced from the parent metal to the HAZ, to the TMAZ and then finally the NZ. The NZ had fine equiaxed grains due to recrystallization. The lowest hardness was seen in the HAZ and the highest hardness was seen in the parent metal. The tensile and the yield strength of the welds were found to be less than the base metal, whilst fracture was found in the HAZ area which had the lowest hardness. However, FSW was found to have improved the corrosion resistance and the HAZ had better corrosion resistance of them all.

Jonckheere et al. [21] studied the mechanical and fracture behaviour of 6063-T6 aluminium alloy friction stir spot welding. In this research, the influence of pin lengths of various dimensions was examined on the spot-welded lap joints. The fracture surfaces were examined critically via macrographic examination. The rotational speed used in this study was $1500 \mathrm{rpm}$, plunge rates of 5 and $12 \mathrm{~mm} / \mathrm{min}$, while different pin lengths were employed such as 3.5, 4.0, 4.5, 5.0 and $5.7 \mathrm{~mm}$. Crack assessment was carried out on the fracture surfaces under type 1-3 fractures. It was concluded that Type 3 fracture should be favoured because it happens outside the weld itself and thus requires substantial plastic deformations and ultimate forces.

\subsection{FSSW of dissimilar alloys}

FSW and FSSW is a vigorous welding technique, which is capable of successfully welding dissimilar and similar alloys. Various tests have been documented on FSSW and FSW of dissimilar alloys. Yeni et al. [1] helped fill the gap by investigating FSSW of AA 1050 and pure $\mathrm{Cu}$ where the plunged depth was varied. The authors concluded that the hardness of $\mathrm{Cu}$ was affected by the plunge depth, as the depth of plunging increases the heat generated rises resulting in the grain size increasing thus decreasing the hardness of the alloy.

Chen et al. [22] focused on comparing AA 6061 and advanced high strength steel varying the plunge rate and the dwell time. It was concluded that a large plunge from the high dwell time formed preliminary cracks in the intermetallic compounds which can be studied as weld defects. Fereiduni et al. [23] concentrated on the effect of the microstructure observation with its intermetallic phases and joint interface and the dwell time in order to describe the variance of weld strength, conducting FSSW on AA 5083 and steel-12 sheets. The dwell time was varied and was seen to produce stronger joints. As it increased, the thickness of the intermetallic phases produced increased, changing the morphology of the materials, which can further be concluded that the dwell time influences the weld strength, in this case, the strength increased as the dwell time increased.

Bozkurt et al. [2] studied how varying the dwell time, the tilt angle and the tool plunge depth influenced the lap shear tensile properties of dissimilar FSSW materials, when FSSW was conducted on AA 2024-T3 and AA 5754-H22. It was found that welds that generated a low LSFL ruptured with a cross-nugget failure mode and the welds that generated a high LSFL ruptured with a pull-out nugget failure mode. The tool penetration depth increased up to a certain point as the width of the weld nugget increased. As the dwell time increased, it was found that the joint strength increased due to the width of the weld nugget increasing. The tilt angle of the tool is inversely proportional to the joint strength, as it increased the joint strength decreased due to the inconvenient in the stirring. 
The tool design was studied by various authors and a sound conclusion was produced. Ravikumar et al. [24] studied how the process parameters have an impact on the mechanical properties of AA 6061-T651 and AA 7075-T651 conducted from FSW. The tool rotation rate, plunge rate and tool pin were varied. It was found the hardness decreased as the distance to the weld centre decreased. The plunge rate increased as the size of the HAZ decreased. The over the aging of the constituents in the HAZ is determined by on the heat exposure time and the temperature and is controlled by the tool rotation speed and the plunge rate; which are also deciding factor for the hardness distribution in the HAZ.

Abbass et al. [25] attempted to optimise the mechanical properties of FSSW joints for dissimilar aluminium alloys, where FSSW was conducted on AA 2024-T3 and AA 5754-H114. The pin profile, tool rotation rate and dwell time was varied. It was found that the tensile shear force increased as the dwell time increased up to a certain point and then decreased. At a constant pin profile, the shear force increased as the tool rotation rate increased, and lower hardness was found in the HAZ and the TMAZ because of the intermetallic compounds detected by XRD.

Li et al. [26] carried out a study on dissimilar FSSW of AA6063/AA5083. In this study, the influence of sleeve plunging rate on lap welded joints leading to tensile shear properties and microstructures were explored. Results showed that the keyhole can be removed with the use of FSpW. It was noted that, as the plunging rate increased, the bending hooks changed to a flat morphology. Substantial bonding was formed in the sleeve affected zone and at the lap interface. When the plunging speed of the sleeve increased from 40 to $110 \mathrm{~mm} / \mathrm{min}$, the lap shear failure loads for the joints increased first, and then decreased. Using a sleeve plunging speed of $80 \mathrm{~mm}$ a cumulative failure load of $8017 \mathrm{~N}$ was achieved.

Various authors documented tests done by varying different process parameters and using various materials. However, the knowledge of studying the microstructural development, electrochemical integrity, and the mechanical properties of FSSW by varying the tool rotation rate and dwell time on a similar material using AA6063 is limited. Hence, the reason for selecting this material for its application in aerospace, marine and automobile industries.

\section{Experimental procedure}

The FSSW, the experimental design and research methodology used while conducting this research is emphasised on. The design of experiments conducted, the standards, software and various pieces of equipment used to generate data for this research are here elaborated. As the main aim of the work was to study how the evolving properties of friction stir spot welds of AA 6063 are affected by the different process parameters, the FSSW of a conveniently prepared specimen, various metallurgical testing, mechanical properties testing and corrosion testing were conducted.
Table 1. FSSW process parameters.

\begin{tabular}{lll}
\hline Dwell time (s) & Experiments & $\begin{array}{l}\text { Tool rotation } \\
\text { rate (rpm) }\end{array}$ \\
\hline \multirow{3}{*}{10} & 1 & 600 \\
& 2 & 900 \\
15 & 3 & 1200 \\
& 4 & 600 \\
& 5 & 900 \\
& 6 & 1200 \\
\hline
\end{tabular}

\subsection{Friction stir spot welding process parameters}

Commercial aluminium alloy 6063 sheets; with a width of $30 \mathrm{~mm}$ and a thickness of $2 \mathrm{~mm}$ were used for this research. The received sheet materials were sectioned into feasible, easily useable sizes using the abrasive cutting machine and were strategically designed for the intended FSSW process.

The chief parameters involved during the FSSW process include the tool rotation rate and the dwell time and these parameters were used for the investigation of this research work as depicted in Figure 2a. The tool rotation rate used for this research was 600,900 and $1200 \mathrm{rpm}$ and the dwell time used was 10 and $15 \mathrm{~s}$ : as seen in Table 1.

Furthermore, the following process parameters were kept constant to achieve the desired results

- a cylindrical tapered tool pin profile made of AISI H13 steel was used;

$-0.2 \mathrm{~mm}$ plunge depth process parameter was used;

- a $2^{\circ}$ tool tilt angle was used for this research work.

Figure $2 \mathrm{~b}$ depicts the visual observation of the outcome of the fabricated spot welds samples carried out with varying the tool rotation rate as well as the dwell time on the AA6063 in the above-mentioned conditions. The spot welds with distinct keyhole characteristic is the main outcomes. The distinct spot welds produced by the friction stir spot welding process were subjected to various metallographic and mechanical testing such as; phase analysis using the XRD, metallographic examination using the stereo-microscope and the optical microscope, hardness testing using the Micro Vickers Hardness Test, tensile shear testing using the tensile testing equipment and corrosion testing using the Potentiostat corrosion testing machine.

\subsection{Microstructural characterization}

The chemistry obtained for the as-received material and the spot welds were performed using the Optical Emission Spectrometer (OES) and the X-ray Diffraction (XRD). The chemical analysis obtained; performed on the as-received material, with the OES helped in demonstrating the major elements present within the material, which concluded the type and the grade of the material received. The Aluminium Global Method $\left(\mathrm{Al}_{100}\right)$ was used to conduct chemical analysis. This specific method was used 
to determine the major elements present within the standard aluminium material.

The XRD analysis was performed on the as-received material and the spot welds, with a Rigaku Ultima IV machine and a $\mathrm{Cu}-\mathrm{K}_{\alpha}$ cathode, helped to identify the different crystallised phases present within the material, which furthermore confirmed the type of the material received. Samples for the chemical analysis were prepared by grinding the surface of the samples with a 1200 Silicon (SiC) Grit Paper; using water as a medium. The grinding process on the samples was conducted to clean and remove any deep scratches or debris that might have been present on the surface of the samples due to the abrasive cutting. The samples were then placed onto the stage of the OES. The ground sample was placed on to the $2 \mathrm{~mm}$ collimator which was placed into the XRD machine. The mineralogical phases were identified using the Rigaku PDXL Software. Data and results were plotted using the Origin 2018b Software.

The microstructures in the parent material as well as in the spot welds were identified using metallographic techniques. The optical microscope with Olympus software and the AxioVision software was used. The sample surface was etched with Keller's reagent ( $2 \mathrm{ml} \mathrm{HF}, 3 \mathrm{ml} \mathrm{HCl}, 5 \mathrm{ml}$ $\mathrm{HNO}_{3}$ and $190 \mathrm{ml}$ water) [27] to reveal the microstructure in the samples. This specific etchant was used as it is a conventional etchant for Aluminium 6000 series alloy. It also aids in revealing the distinct grain boundaries and microstructural constituents present within the material using the American Society for Testing and Materials (ASTM) metallographic recommended procedures reported in [27].

\subsection{Mechanical properties and corrosion testing}

Hardness testing and tensile shear testing were the mechanical tests conducted for this research study on the as-received material and spot welds. They were selected so that the outcomes therefore inform on the possible areas of applications for this material. The hardness tests were conducted using the Micro-Vickers Hardness testing machine with a diamond indenter. Ten indentations were done per sample. The tensile shear tests were conducted using the tensile testing machine with the TestXpert II software. This specific standard is generally used for shear testing on aluminium spot welds. Tensile shear was conducted on the welded samples in accordance with ASTM E8M-13 standard test methods. The schematic Illustration of tensile shear samples on tensile machine is depicted in Figure 3a while Figure 3b depicts tensile shear specimen showing stress mechanism due to shear stress such that $\Phi \mathrm{d}$ represents diameter, $\tau x$ represents shear stress, $t$ is for thickness while $F$ is for tension-shear i.e. fracture force during FSSW, and lastly Figure 3c presented sample of the spot-welded material [28]. The tensile shear strength was tested. It provided the maximum shear tensile strength of the aluminium 6063 alloys. The equipment used additionally displayed a useful stress-strain graph.

Furthermore, the additional and significant material property was selected to obtain a representative trend on how the material behaves in a corrosive environment.
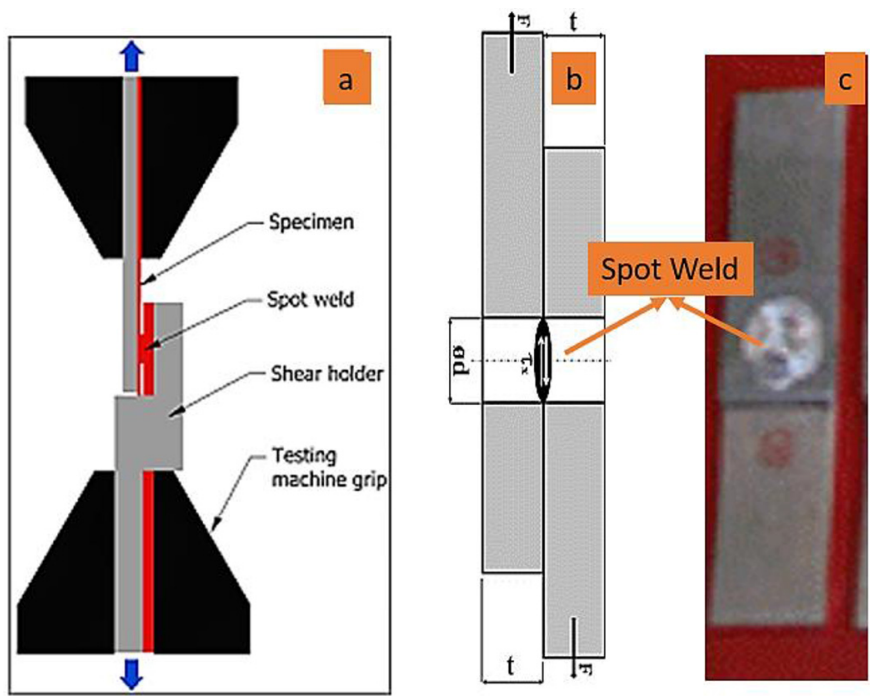

Fig. 3. (a) The schematic illustration of the tensile shear on the tensile machine (b) Joints lap configuration of FSSW specimen for tensile shear test (c) Sample prepared for tensile shear testing.

The corrosion testing obtained was conducted using the Potentiostat Corrosion testing machine. The corrosion test was conducted using Artificial Seawater (ASW) as a medium $\left(30 \mathrm{~g} \mathrm{NaCl}, 0.8 \mathrm{~g} \mathrm{KCl}, 6.6 \mathrm{~g} \mathrm{MgSO}_{4}, 0.5 \mathrm{~g} \mathrm{NaHCO}_{3}\right.$ and $1.3 \mathrm{~g} \mathrm{CaCl}_{2}$ ) [29]. This medium was selected because aluminium alloy 6063 is popular in marine applications, moreover; ASW is an ideal representative of the marine life. The DY2322 Instrument Model made by Digi-Ivy Potentiostat corrosion machine was used with the Linear Voltaic (LV) program. This corrosion testing aided in representing how corrosion resistant the material is to an ASW. The sample prepared for corrosion testing using the Potentiostat was prepared by cold mounting the sample in a rubber mould using cold mounting resin; attaching the sample to a copper wire, leaving the required surface exposed as seen in Figure 4. The mounted sample was ground using 1200 silicon ( $\mathrm{SiC}$ ) Grit Paper to clean and remove any debris that might have been present on the surface of the sample. The sample was then placed inside the ASW medium. The Potentiostat corrosion test aided in identifying the corrosion rates of the as-received material as well as also identifying the Tafel plots for the as-received material; which helps us to know ideally when corrosion might attack the material and also to know which reactions are most dominant further help in protecting the material. The following parameters were used for the Potentiostat corrosion testing; the potential difference from -2 (minus) to +2 (positive), the scan rate $0.05 \mathrm{~V} / \mathrm{s}$ and the sensitivity is $1.0 \mathrm{e}^{-3}$

\section{Results and discussion}

This research work was developed to understand how the evolving microstructural analysis and mechanical properties of FSSW-based spot welds were affected under various process parameters. Results were produced and 
documented through diverse tests. The recent section describes the outcomes and data produced from the experimental design used in this analysis. The results obtained include chemical analysis, metallography testing, XRD analysis, hardness testing, corrosion testing of potentiostats and tensile shear testing are discussed below.

\subsection{Metallography examination}

The surface appearances of the welds produced by friction stir spot welding of AA6063 were observed on the spot weld generated on the overlapping aluminium sheets. The macrostructures of the sample were analysed with an analysis software equipped stereo microscope with $5000 \times$ magnification. It is noticed, Figure 5, that the tool rotation rate and the dwell time both influences the surface appearance or morphology of the spot welds. The stereo microscopic examination of the welds at $500 \times$ magnification shows different macrostructures of the spot welds as depicted in Figure 5. The macrostructures of the spot welds obtained at $5000 \times$ magnification.
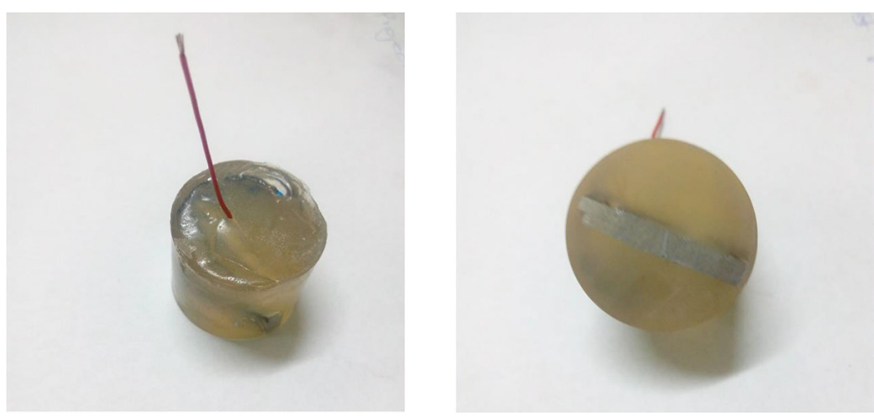

Fig. 4. Samples prepared for the Potentiostat corrosion testing.
From the stereo microscopic examination, it is noticed that the spot welds are shown with distinct keyholes at the centre of the $2 \mathrm{~mm}$ thick overlapping aluminium sheets. The observed keyholes were produced by the tapered cylindrical pin tool as the friction stir spot welding proceeded. Figure 5 shows more aluminium debris generated when the tool rotates at $600 \mathrm{rpm}$ at a fixed dwell time. The keyhole cleans up i.e. has fewer aluminium debris as the tool rotational rate increases to $1200 \mathrm{rpm}$ for fixed dwell time.

One can observe that the rotational tool rate and the dwell time have an impact on the weld surfaces obtained. Furthermore, as the tool rotational rate increases at a fixed dwell time (at $10 \mathrm{~s}$ ) it is observed that some debris seem to appear at $600 \mathrm{rpm}$, less debris appears at $900 \mathrm{rpm}$ and at $1200 \mathrm{rpm}$ it observed that no debris appeared; a smooth and clear spot weld was produced. At a constant dwell time of $15 \mathrm{~s}$, it was observed that debris appears at $600 \mathrm{rpm}$, less debris appears at $900 \mathrm{rpm}$ and at $1200 \mathrm{rpm}$ it was seen that no debris appears; a smooth, clear and fractured spot weld was produced. It can further be seen that as the dwell time increases; at a constant tool rotation rate less debris was produced, a smooth, clear spot weld surface and fractured spot weld formed. It is reassuring to conclude that as the tool rotation rate increases; at a constant dwell time a smoother and clearer spot weld surface will form.

\subsection{Microstructural examination}

The distinct microstructural features: being the heataffected zone (HAZ), thermo-mechanically affected zone (TMAZ) and the stir zone (SZ) obtained from the microstructural analysis can be seen in Figure 6. The spot welds were etched with the 1 A reagent $(100 \mathrm{ml}$ water and
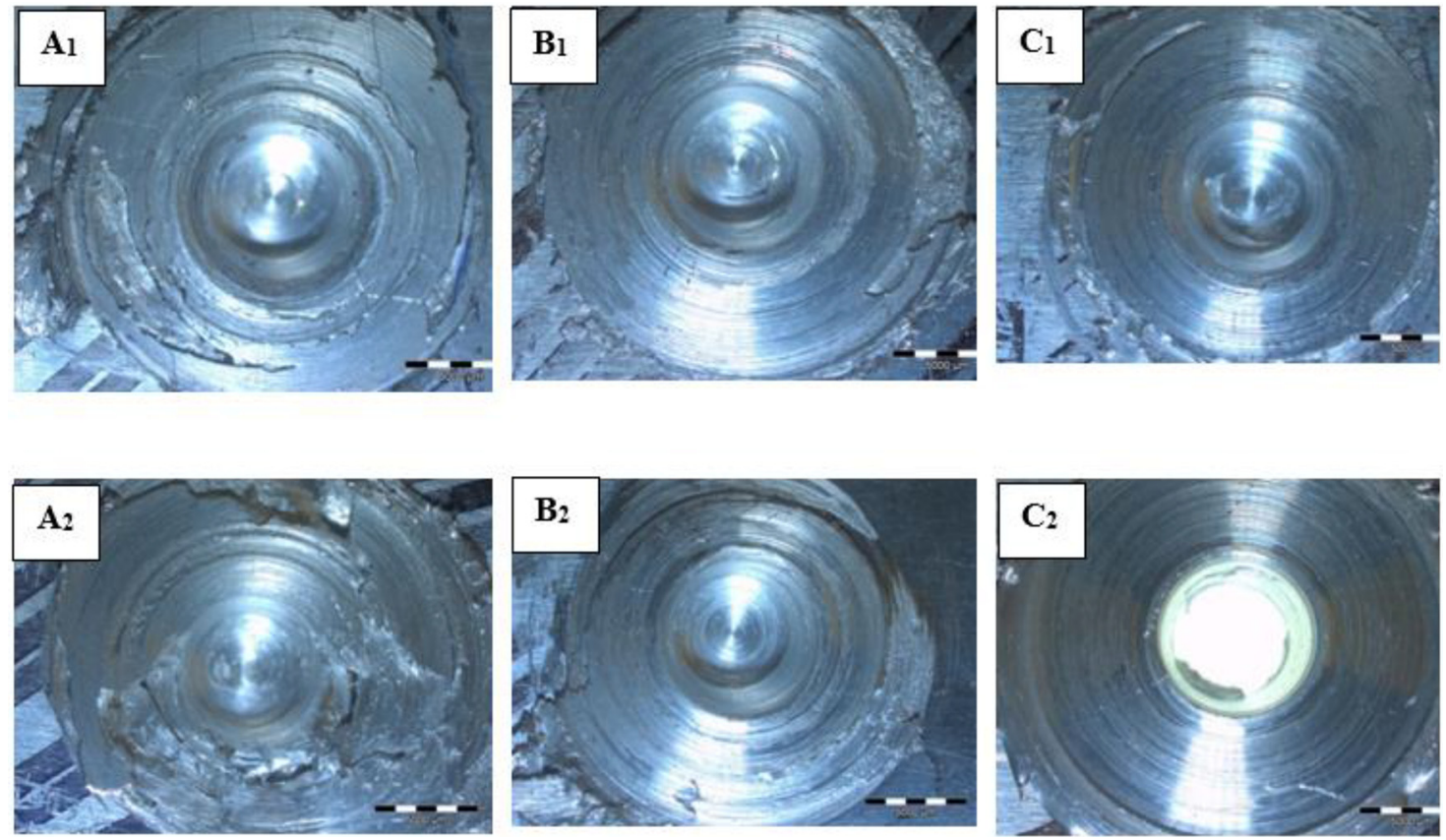

Fig. 5. Macrostructure of the weld produced with FSSW; $\mathrm{A}_{1}: 600 \mathrm{rpm}$ at $10 \mathrm{~s}, \mathrm{~B}_{1}: 900 \mathrm{rpm}$ at $10 \mathrm{~s}, \mathrm{C}_{1}: 1200 \mathrm{rpm}$ at $10 \mathrm{~s}, \mathrm{~A}_{2}: 600 \mathrm{rpm}$ at $15 \mathrm{~s}, \mathrm{~B}_{2}: 900 \mathrm{rpm}$ at $15 \mathrm{~s}, \mathrm{C}_{2}: 1200 \mathrm{rpm}$ at $15 \mathrm{~s}$. 


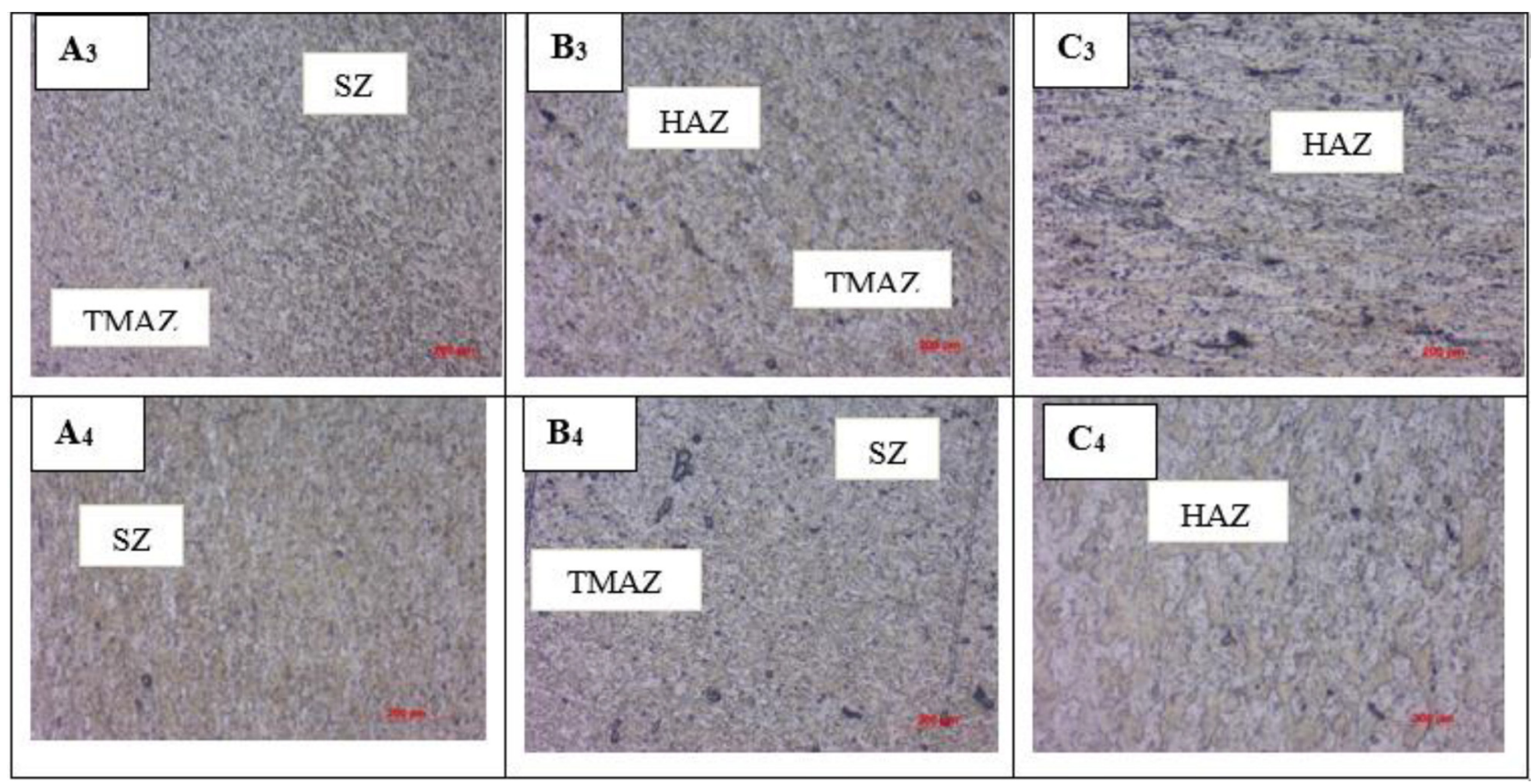

Fig. 6. The microstructure of the spot weld produced at $10 \mathrm{~s} ; \mathrm{A}_{3}: 600 \mathrm{rpm}$ at $10 \mathrm{~s}, \mathrm{~B}_{3}: 900 \mathrm{rpm}$ at $10 \mathrm{~s}, \mathrm{C}_{3}: 1200 \mathrm{rpm}$ at $10 \mathrm{~s} \mathrm{~A}_{4}: 600 \mathrm{rpm}$ at $15 \mathrm{~s}, \mathrm{~B}_{4}: 900 \mathrm{rpm}$ at $15 \mathrm{~s}, \mathrm{C}_{4}: 1200 \mathrm{rpm}$ at $15 \mathrm{~s}$ (at $200 \times$ magnification).

$1 \mathrm{ml} \mathrm{HF}$ ) [27] and were obtained using the optical microscope using the AxioVision software at $50 \times$ and $200 \times$ magnification as seen in Figure 6 .

The microstructural analysis for the spot welds produced at $10 \mathrm{~s}$ is represented in Figure 6 with a magnification of $200 \times$. The rotational tool rate and the dwell time have significantly affected the microstructures; three (3) microstructural evolutions are shown; namely the SZ, the HAZ and the TMAZ $[18,20,30]$.

The SZ shows recrystallized equiaxed grains which are smaller than in the as-received material; which correlates to the hardness property, having the highest hardness $[23,17]$. The HAZ has coarse grains due to the frictional heat applied and which is generally found closer to the weld centre and has black characteristics of dark dots [31] in the microstructure, having the lowest hardness [17,23]. The TMAZ has small grains and appears similar to the parent material and the BM represents the base metal.

Although, Jambhale et al. [32] suggested that there is no clear correlation between the tool rotation rate and the microstructural evolution, as the tool rotation rate increases at a constant dwell time (at 10s), more SZ develops in the microstructure (from 600 to $900 \mathrm{rpm}$ ), hence finer grains develop; contrary to the HAZ developed at $1200 \mathrm{rpm}$, hence the coarse grains develop. The microstructural analysis for the spot welds produced at $15 \mathrm{~s}$ is represented in Figure 6 with a magnification of $200 \times$. As the tool rotation rate increases at a constant dwell time (at 15 s) more TMAZ develops in the microstructure (from 600 to $900 \mathrm{rpm}$ ), hence the hardness increases as well, similar to the dwell time at $10 \mathrm{~s} \mathrm{HAZ} \mathrm{develops} \mathrm{at} 1200 \mathrm{rpm}$, hence the hardness decreases. Furthermore, as the dwell time increases at a constant tool rotation rate at $600 \mathrm{rpm}$ more SZ develops, at $900 \mathrm{rpm}$ the SZ and the TMAZ is shown and at $1200 \mathrm{rpm}$ the HAZ can only be observed.
The microstructural evolution at $200 \times$ magnification shows that as the tool rotation rate increases at a constant dwell time (at $10 \mathrm{~s}$ ) the HAZ develops then the TMAZ further develops from 600 to $900 \mathrm{rpm}$ then finally the HAZ develops even more at $1200 \mathrm{rpm}$, hence coarse grains were seen. As the tool rotation rate increases at a constant dwell time (at $15 \mathrm{~s}$ ) the HAZ and the TMAZ develops from 600 to $900 \mathrm{rpm}$ then finally the HAZ develops even more at $1200 \mathrm{rpm}$, hence coarse grains were well. Furthermore, as the dwell time increases at a constant tool rotation rate at $600 \mathrm{rpm}$ more SZ develops, at $900 \mathrm{rpm}$ the SZ and the TMAZ is shown and at $1200 \mathrm{rpm}$ the HAZ can be observed, hence coarse grains can be seen.

It is safe to say that as the tool rotation rate increases at a constant dwell time, the HAZ tends to develop gradually hence coarse grains begins to develop. Furthermore, as the dwell time increases at a constant tool rotation rate, finer equiaxed grains were seen at 600 and $900 \mathrm{rpm}$ and finally coarse grains were developed at $1200 \mathrm{rpm}$. The tool rotation rate and the dwell time are some of the factors that led to heat generation during the FSSW process and increasing (about the different FSSW parameters delegated for this study); more heat is applied, thus changing the microstructural modes evidently changing the integrity of the material.

Table 2 and Figure 7 present and display respectively the percentages chemical composition and energy dispersive spectra of the welds at varying tool rotational rates and dwell times. It is noticed at lower tool rotational rate and a higher dwell time i.e. $15 \mathrm{~s}$ the amount of retained aluminium materials in the keyhole increases with an increase of tool rotational rate from 600 to $1200 \mathrm{rpm}$. This observation corroborates with the stereo microscopic findings that at higher tool rotational rate the keyhole is much smoother hence having more aluminium materials 
Table 2. Chemical composition at the welds as measured with energy dispersive spectroscopy. It is in the function of tool rotational rate and dwells time.

\begin{tabular}{|c|c|c|c|c|c|c|c|c|c|c|c|c|c|}
\hline & $\mathrm{C}$ & $\mathrm{N}$ & $\mathrm{O}$ & $\mathrm{F}$ & $\mathrm{Na}$ & $\mathrm{Al}$ & $\mathrm{Si}$ & $\mathrm{S}$ & $\mathrm{Cl}$ & K & $\mathrm{Ca}$ & $\mathrm{Fe}$ & $\mathrm{Ag}$ \\
\hline $600 \mathrm{rpm}, 10 \mathrm{~s}$ & 9 & & 1.5 & 2 & & 86 & & & & & & 0.20 & 1.30 \\
\hline $900 \mathrm{rpm}, 10 \mathrm{~s}$ & 5.24 & 5.1 & 28.07 & 1.89 & & 58.51 & & & 0.10 & & & 0.14 & 0.95 \\
\hline $1200 \mathrm{rpm}, 10 \mathrm{~s}$ & 8.80 & & 6.17 & 7.37 & & 76.36 & & & & & & 0.25 & 1.05 \\
\hline $600 \mathrm{rpm} ; 15 \mathrm{~s}$ & 68 & 2.4 & 14 & 1 & 0.2 & 12 & 0.43 & 0.35 & 0.30 & & 0.44 & 0.36 & \\
\hline $900 \mathrm{rpm} ; 15 \mathrm{~s}$ & 3.27 & 7.47 & 50.34 & 5.10 & & 33.21 & & & 0.43 & & & 0.18 & \\
\hline $1200 \mathrm{rpm} ; 15 \mathrm{~s}$ & 9.49 & & 2.33 & 2.58 & & 84.12 & & & & & & 0.23 & 1.25 \\
\hline
\end{tabular}
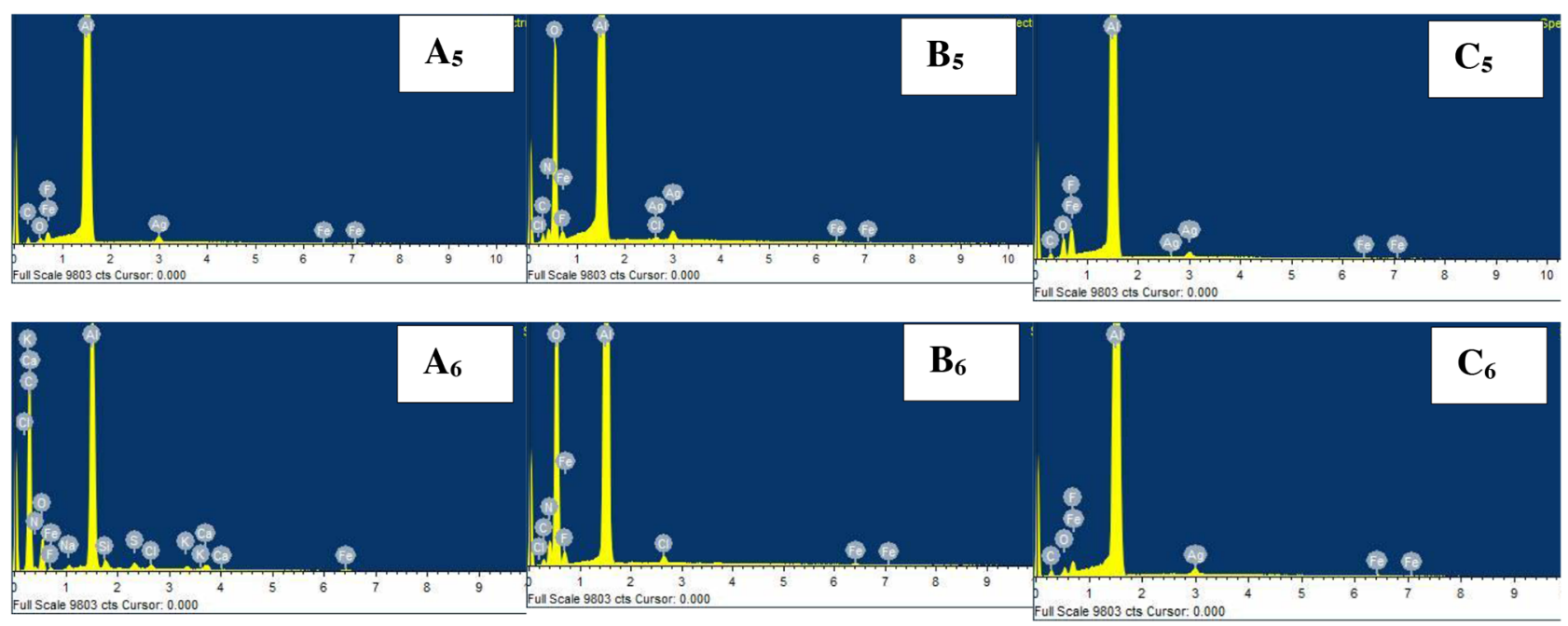

Fig. 7. Energy dispersion spectroscopy spectra and chemical composition of the weld produced at different tool rotational rate and dwell times. $\mathrm{A}_{5}: 600 \mathrm{rpm}$ at $10 \mathrm{~s}, \mathrm{~B}_{5}: 900 \mathrm{rpm}$ at $10 \mathrm{~s}, \mathrm{C}_{5}: 1200 \mathrm{rpm}$ at $10 \mathrm{~s}, \mathrm{~A}_{6}: 600 \mathrm{rpm}$ at $15 \mathrm{~s}, \mathrm{~B}_{6}: 900 \mathrm{rpm}$ at $15 \mathrm{~s}, \mathrm{C}_{6}: 1200 \mathrm{rpm}$ at $15 \mathrm{~s}$.

$(84.12 \%)$ than in the lower rotational rate whose keyhole was found shattered hence leaving only a small amount of aluminium $(12 \%)$ in the keyhole. The above observation was not as obvious as in the case of a lower tool rotational rate $(600 \mathrm{rpm})$. The energy dispersion for the spot welds produced from the EDS can be seen in Figure 7.

\subsection{Chemical analysis}

The optical emission spectrometer (OES) was used to determine the chemical composition of all the materials used i.e. the parent as received as well as the welds. The chemical composition of the as-received AA6063 material as analysed with the OES, Table 3 was compared to the documented standard of AA6063 as shown in Table 4.

The chemical analysis obtained for the as-received AA6063 material was shown in Table 3 and the chemical analysis obtained for a standard AA6063 obtained from the AA60603 datasheet from the Aerospace Specification Metals Inc. (ASM) [33] was shown in Table 4. The chemical analysis from the as-received material falls within the specification range of the standard material Aluminium Alloy 6063-T4, which further concludes that the material received is indeed a standard AA6063 material.
Table 3. Results obtained from the Chemical analysis of the as-received AA6063 material.

\begin{tabular}{llll}
\hline $\mathrm{Mg}$ & $\mathrm{Si}$ & $\mathrm{Fe}$ & $\mathrm{Al}$ \\
\hline $0.493 \%$ & $0.395 \%$ & $0.199 \%$ & $98.82 \%$ \\
\hline
\end{tabular}

Table 4. The chemical analysis obtained for a standard AA6063 material based on the AA6063 datasheet [33].

Chemical Analysis for standard AA 6063 material

\begin{tabular}{llll}
\hline $\mathrm{Mg}$ & $\mathrm{Si}$ & $\mathrm{Fe}$ & $\mathrm{Al}$ \\
$0.45-0.9 \%$ & $0.20-0.60 \%$ & $0.00-0.35 \%$ & Balance \\
\hline
\end{tabular}

Presenting the oxygen - aluminium ratio at the welds in the function of tool rotational rate and dwell time in Figure 8, one notices oxygen uptake by the keyhole reaches a peak at around $900 \mathrm{rpm}$ tool rotational rate before declining. This might indicate the formation of a thin oxide layer on the keyhole as demonstrated by an increased oxygen amount up to $28.07 \%$ and $50.34 \%$ for $900 \mathrm{rpm}$ at 


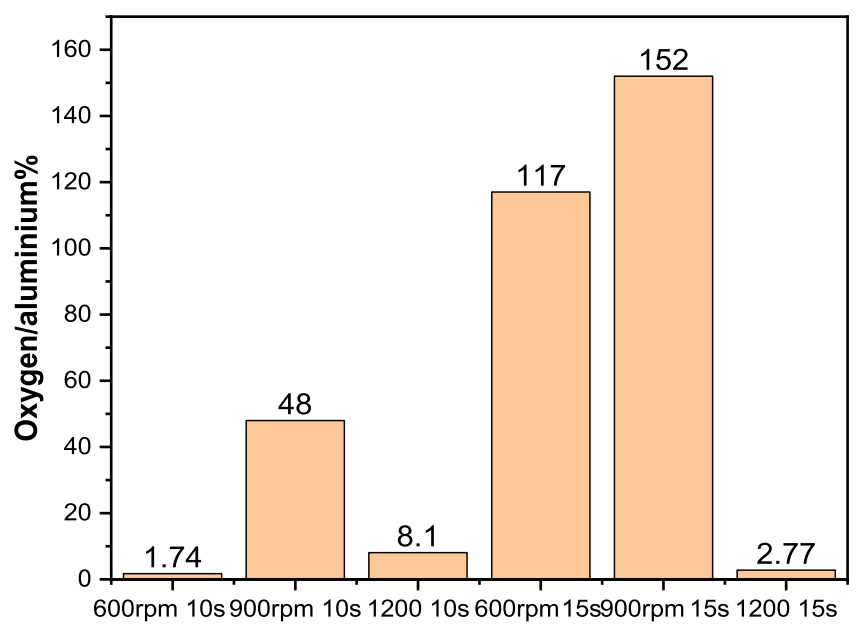

Process parameters

Fig. 8. Oxygen- aluminium ratio $\%$ at the welds in the function of tool rotational rate and dwell time.

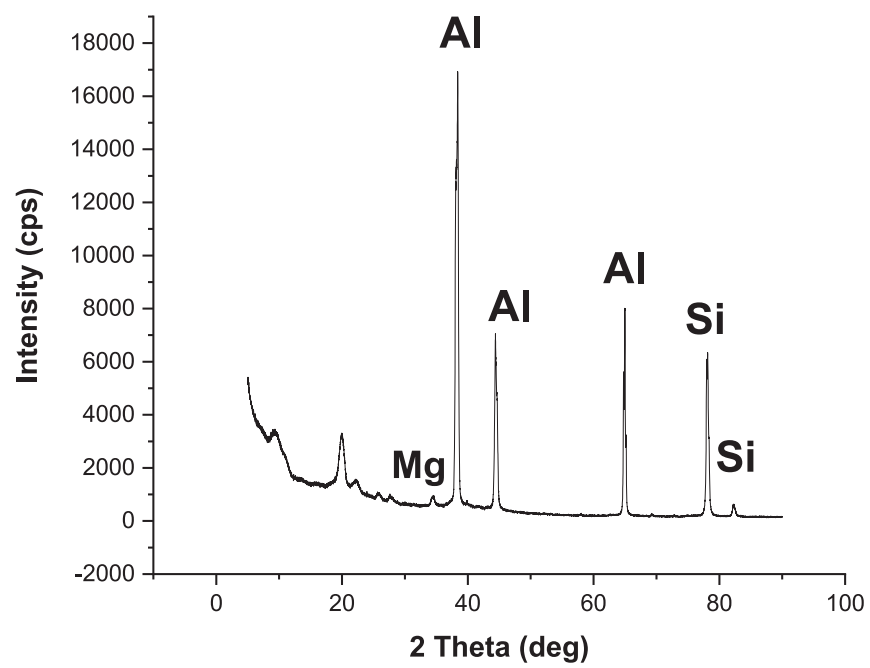

Fig. 9. The XRD analysis for the as-received AA6063 material.

$10 \mathrm{~s}$ and $900 \mathrm{rpm}$ at $15 \mathrm{~s}$ respectively. This oxide layer might prevent further oxygen uptake.

\subsection{Structural characteristics: XRD analysis}

The XRD analysis was obtained using the Rigaku Ultima IV X-Ray Diffraction equipment with the Rigaku PDXL Software using a $2 \mathrm{~mm}$ collimator and was represented using the OriginPro 2018b Software. An XRD analysis for the as-received AA6063 material is shown in Figure 9.

X-ray Diffraction (XRD) analysis aids in demonstrating and studying the typical phases present within a given material. The XRD pattern for the as-received AA 6063 material displayed in Figure 9 demonstrates a typical pattern for an aluminium material. The highest peak is found around 40 two-Theta (deg) which is similar to a standard aluminium material as supported by Atuanya et al. [34]. Furthermore, the XRD pattern represented has characteristics phases of $\mathrm{Al}, \mathrm{Mg}$ and $\mathrm{Si}$, which further confirms that the material at hand is an aluminium 6000 series alloy. XRD diffractograms of the spot welds produced by friction stir spot welding of AA6063 specimen is shown in Figure 10.

The tool rotation rate, as well as the dwell time, had a significant effect on the XRD analysis. A modification of the XRD pattern especially around $2 \theta=40^{\circ}$ has been observed as the tool rotation rate increases from 600 to $1200 \mathrm{rpm}$ at a constant dwell time of $10 \mathrm{~s}$. Similar to the asreceived material, intermetallic phases of Si and aluminium magnesium AlMg might be present. As the tool rotation rate increases, the XRD patterns display the presence of more intermetallics revealing the stir zone (SZ) formation of SZ formation is identified as it is shown that at around 40 two-Theta (deg) more intermetallic phases of AlMg is seen to form, which might be associated to the SZ formation as seen in the microstructural evolution previously documented. On further processing, more intermetallic phases of $\mathrm{AlMg}$ forms as the tool rotation rate increases from 600 to $900 \mathrm{rpm}$ which might be connected to the increase in the SZ formation however at $1200 \mathrm{rpm} \mathrm{Si}$ is present, which might be connected to the increase in the HAZ formed on welding. Moreover, a change in the XRD pattern can be seen as well at a constant $15 \mathrm{~s}$ dwell time. As the tool rotation rate increase it is shown that at around 40 twoTheta (deg) more intermetallic phases of Si is seen to form, which might be associated to the TMAZ formation presented in the microstructural analysis. On further processing, similar to the constant dwell time at $10 \mathrm{~s}$, more intermetallic phases of $\mathrm{AlMg}$ forms as the tool rotation rate increases from 600 to $900 \mathrm{rpm}$, however, it might be connected to the SZ increasing and the development the TMAZ; nevertheless at $1200 \mathrm{rpm} \mathrm{Si}$ is present, which might be connected to the increase in the HAZ formed on welding.

At $600 \mathrm{rpm}$ some slight variations can be seen in the XRD pattern as the dwell time increases. It can be seen that at around 40 two-Theta (deg), more intermetallic phases of $\mathrm{AlMg}$ is seen to form. On further processing, more intermetallic phases of Si forms as the dwell time increases, due to an increase in the temperature which might be connected to the increase in the material-tool interactions, hence the increase in the SZ formation. At $900 \mathrm{rpm}$ some slight variations can be seen in the XRD pattern as the dwell time increases. It can be seen that at around 40 twoTheta (deg) slight intermetallic phases of Si was found to have formed but mostly $\mathrm{AlMg}$ was seen to have formed. On further processing, more intermetallic phases of Si forms as the dwell time increases which might be connected to the increase in the SZ and TMAZ which was seen to have formed.

At $1200 \mathrm{rpm}$ some slight variations can be seen in the XRD pattern as the dwell time increases. Similar at $900 \mathrm{rpm}$ it was found that at around 40 two-Theta (deg) slight intermetallic phases of $\mathrm{Si}$ was found to have formed but mostly AlMg was seen to have formed. On further processing, more intermetallic phases of AlMgforms as the dwell time increases which might be connected to the increase in the HAZ which was seen to have formed. 

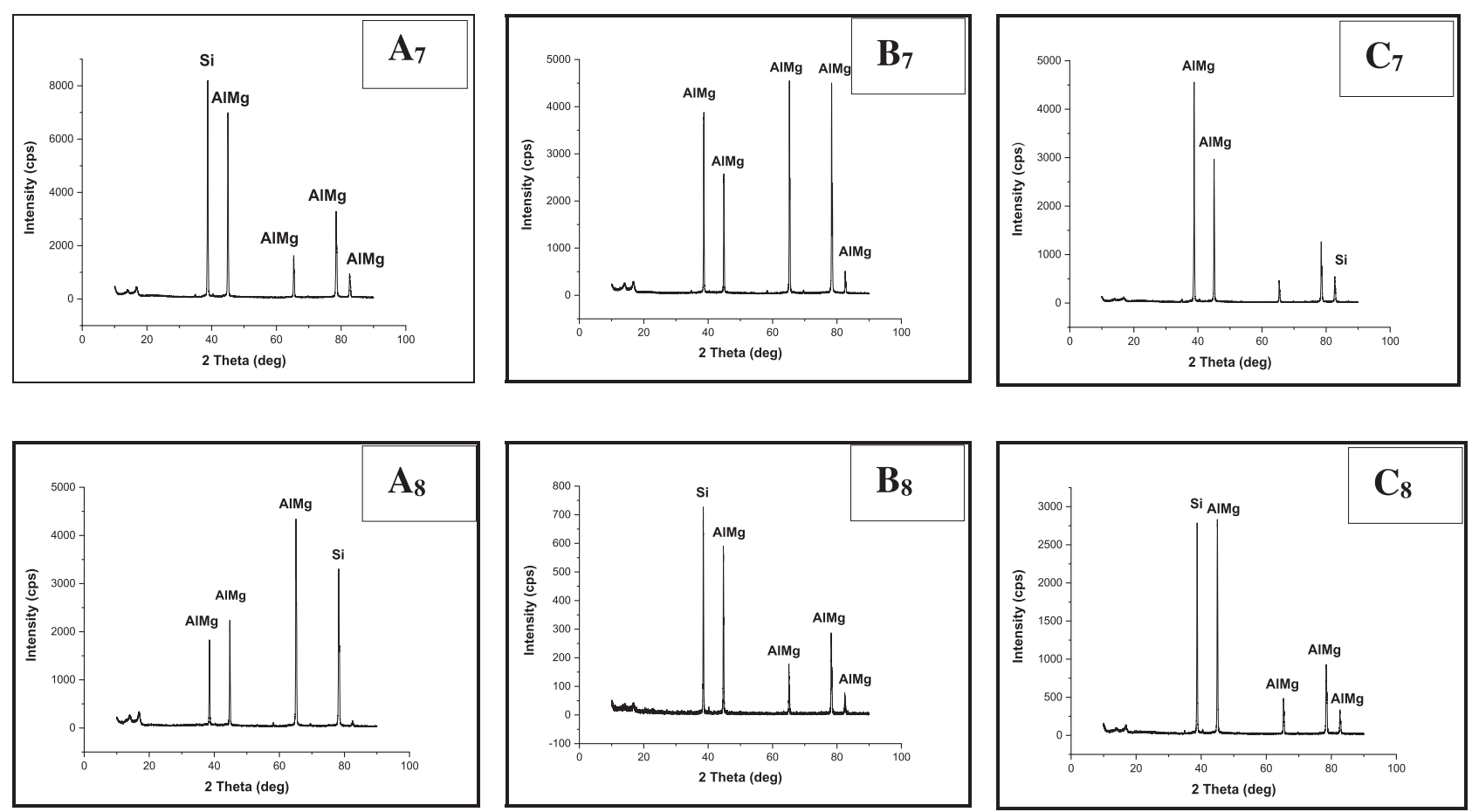

Fig. 10. XRD results of the weld produced with FSSW; $A_{7}: 600 \mathrm{rpm}$ at $10 \mathrm{~s}, \mathrm{~B}_{7}: 900 \mathrm{rpm}$ at $10 \mathrm{~s}, \mathrm{C}_{7}: 1200 \mathrm{rpm}$ at $10 \mathrm{~s}, \mathrm{~A}_{8}: 600 \mathrm{rpm}$ at $15 \mathrm{~s}, \mathrm{~B}_{8}: 900 \mathrm{rpm}$ at $15 \mathrm{~s}, \mathrm{C}_{8}: 1200 \mathrm{rpm}$ at $10 \mathrm{~s}$.

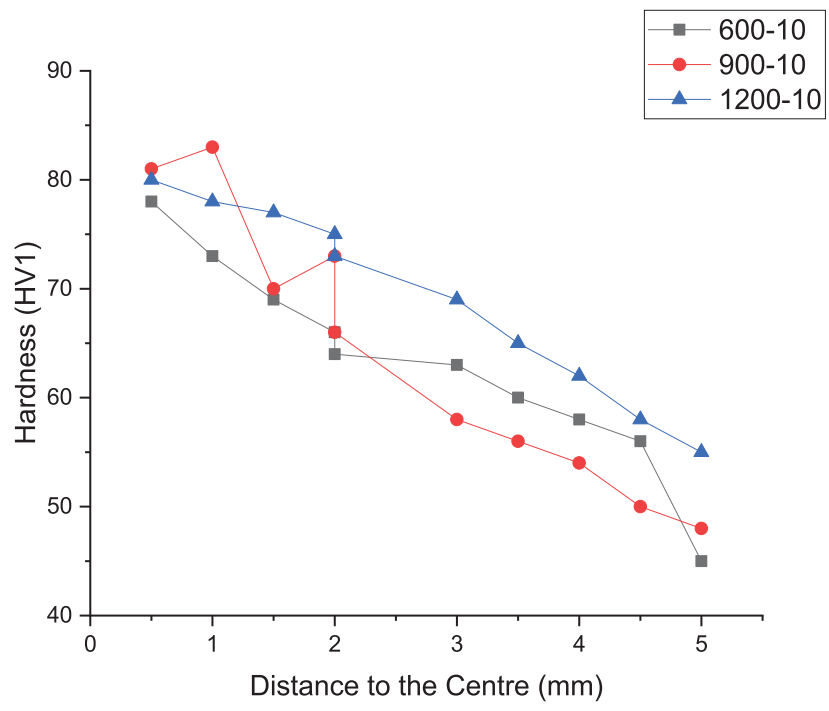

(a)

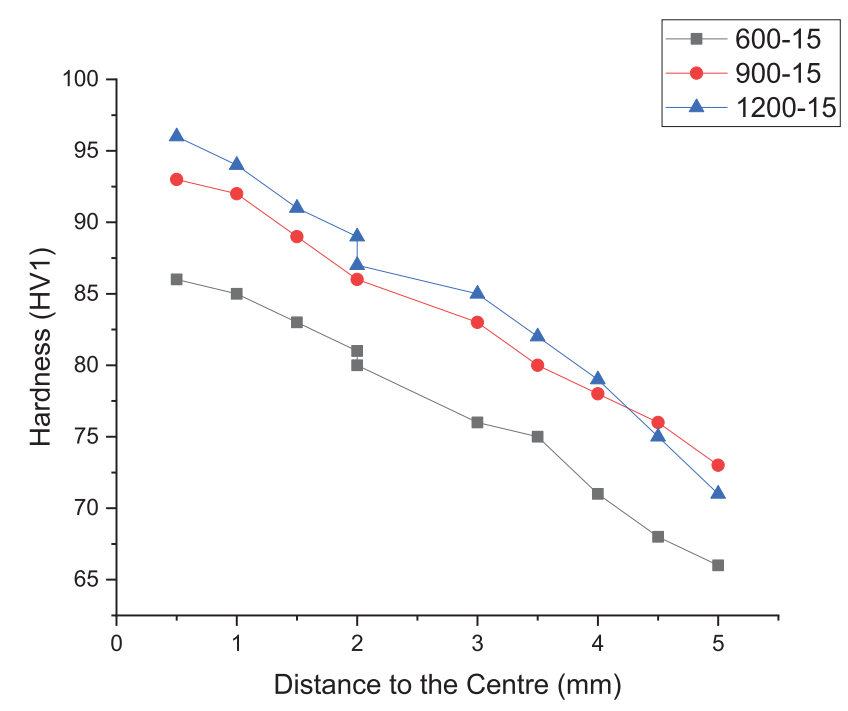

(b)

Fig. 11. (a) The hardness profile for the spot welds produced by FSSW at $10 \mathrm{~s}$ and (b) The hardness profile for the spot welds produced by FSSW at $15 \mathrm{~s}$.

\subsection{Hardness testing}

The hardness values for the as-received material as well as for the spot welds were obtained using the Vickers Hardness testing equipment with a scale of $\mathrm{HV}_{1}$. The hardness value obtained from the Vickers hardness testing machine for the as-received $2 \mathrm{~mm} \mathrm{AA} 6063$ material was 44, $5 \mathrm{HV}_{1}$, which is found to be a standard hardness reading for a $2 \mathrm{~mm}$ thick AA 6063 material. Hardness, amongst others, is a key mechanical property which allows a material to resist penetration, scratching, and plastic deformation. Furthermore, it helps to decide whether the treatment of the material is suitable for the purpose required. The hardness values for the spot welds produced are seen in Figure 11a and 11b.

Fereiduni et al. [23] determined how the dwell time and the microstructural observations of FSSW of AA 5083 and steel -12 sheets are affected. Hamzah et al. [17] studied how the pin shape and the rotation speed affected the mechanical properties and microstructural evolution of 
FSSW 6061. These authors mutually concluded that the hardest region in the microstructural analysis is in the SZ and the softest region is the HAZ, the decrease in the hardness is due to the friction heat and the amount of plastic deformation.

The hardness profile of the spot welds produced by FSSW was seen in Figure 11a and 11b. It can be seen that the results correspond to these authors; the highest hardness profile falls within the SZ region. The hardness was conducted towards the left and right of the FSSW joint. One can see that in both of the dwelling time instances; from the left of the weld towards the joint, the hardness increases then further decreases towards the right of the weld joint. This can be correlated to the formation of the sir zones. At constant dwell time (of $10 \mathrm{~s}$ and $15 \mathrm{~s}$ ) towards the centre on the left, more SZ was present which according to theory shows the highest hardness value [35]. The slight decrease in the hardness towards the right-hand side of the weld joint is shown by the formation of the SZ and the TMAZ. Then gradually the hardness decreases further correlating to the formation of the HAZ present.

The decrease in the hardness profile found is due to the frictional heat on welding and the amount of plastic deformation formed [24]. Regrettably, as the tool rotation rate increases more frictional heat is formed to produce the spot weld; hence with $2 \mathrm{~mm}$ thick sheets results in an inconsistent or a flawed spot weld as well as results obtaining from that weld.

\subsection{Electrochemical characteristics: potentiostat corrosion testing}

AA6063 has many applications including its utilisation in the marine industry. To represent marine applications, corrosion testing was carried out in a corrosive medium with artificial seawater. The corrosion tests were carried out using the Potentiostat and artificial seawater (ASW) $\left(30 \mathrm{~g} \mathrm{NaCl}, 0.8 \mathrm{~g} \mathrm{KCl}, 6.6 \mathrm{~g} \mathrm{MgSO}_{4}, 0.5 \mathrm{~g} \mathrm{NaHCO}_{3}\right.$ and $1.3 \mathrm{~g}$ $\mathrm{CaCl}_{2}$ ) as a medium [29].

The corrosion tests conducted using the Potentiostat led to the determination of the corrosion rate on aluminium samples used. They generated Tafel plots of the spot welds by the linear Voltaic system (LV). The corrosion rate of a metal is the speed at which the metal depreciates in a given environment. It depends on the environment and the metal used [36]. The determination of the corrosion rate helps in determining the lifespan of the material. It may also be used in the selection of the material. In any material, the size of the grains present in the sample affects the corrosion rate of the sample. Finer grains have been found improving the strain hardening of the material, hence promoting the formation of a passive layer, thus lowering the corrosion rate of the material. This ensures that corrosion failure occurs only gradually and over time. On the other hand, coarser grains promote the formation of a lower passive film which increases the corrosion rate; hence this process ensures that the corrosion failure will occur rather rapidly [37].

The corrosion rate for the as-received material was found to be $201.5 \mathrm{~mm}$ per year $(\mathrm{mm} / \mathrm{yr})$. The corrosion rate for the spot welds generated is represented by Figure 12 .

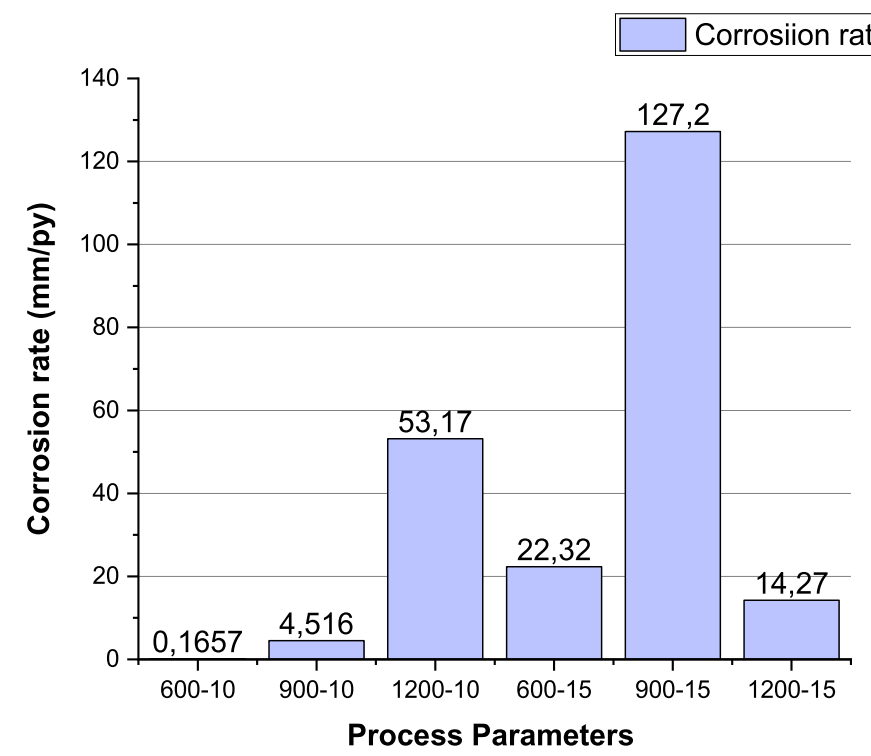

Fig. 12. Corrosion rate for the spot welds.

It is observed that the tool rotation rate, as well as the dwell time, influences the corrosion rate of the welds. As the tool rotation rate increases from 600 to $1200 \mathrm{rpm}$ at a constant dwell time (at $10 \mathrm{~s}$ ), the corrosion rate was found increasing to $53.17 \mathrm{~mm} / \mathrm{yr}$. In agreement with Vuherer et al. [38], the formation of coarser grains was observed around the HAZ, Figure 6. As the rotational tool rate increases at a constant dwell time of $15 \mathrm{~s}$, it was seen that the corrosion rate increases and then further decreases. This outlook disregards the study obtained by Obayi et al. [37]. This viewpoint may be because the effective dwell time to use is $15 \mathrm{~s}$ as a more corrosive sound weld will be produced from 900 to $1200 \mathrm{rpm}$.

The dwell time plays a role in the corrosion rate. It was seen that as the dwell time increases at a constant tool rotation rate of $600 \mathrm{rpm}$ and at $900 \mathrm{rpm}$ the corrosion rate increases; which means that the lifespan of the material will fail quickly As the dwell time increases at $1200 \mathrm{rpm}$ the corrosion rate decreases which concludes that the material will fail gradually over time; which in turn means that the material is more corrosion resistant.

The polarization process displays how a material undergoes a potential change from a stable state. The Tafel plot obtained from the potentiostat polarization test was obtained by the Linear Voltaic (LV) program which further elaborates on the corrosion rates of the material. Furthermore, it presents the nature of the dominant reactions, the anodic arms against the cathodic arms, from this information one, can deduce the method of the impressed current protection: be it anodic protection or cathodic protection. The Tafel plot for the as-received AA6063 material and the produced spot welds at $15 \mathrm{~s}$ dwell time is shown in Figure 13.

An assumption can be made for the Tafel plots of the asreceived as well as the spot welds. The as-received material, as seen in Figure 13 shows a stable Tafel plot having a more cathodic reaction. The spot welds with a rotational tool speed of 600 and $1200 \mathrm{rpm}$ with a dwell time of $15 \mathrm{~s}$ showed 


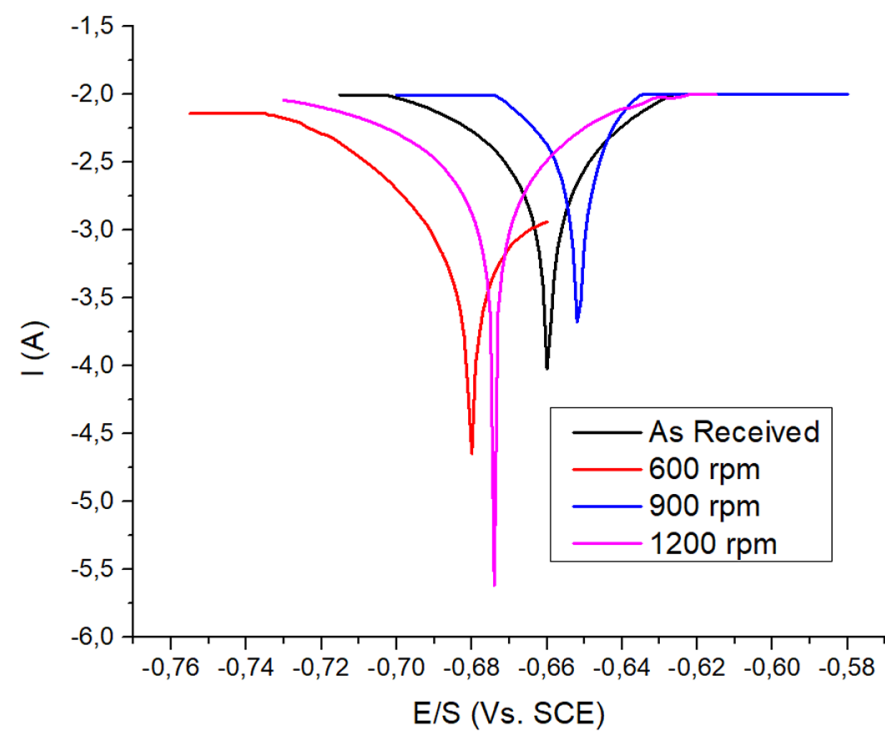

Fig. 13. The Tafel plot for the as-received AA6063 material and the produced spot welds at $15 \mathrm{~s}$ dwell time.

as well, a more cathodic reaction where cathodic protection should be used. However, the Tafel plot for the spot weld having a tool rotation speed of $1200 \mathrm{rpm}$ at a $15 \mathrm{~s}$ dwell time shows a more stable plot having a more cathodic reaction, indicating that more cathodic protection should be used. It can be seen that as the tool rotation rate increase a more anodic reaction occurs, where an anodic reaction is shown to help corrode a material; hence a high corrosion rate was seen and this is consonant with the work reported in [39].

The spot weld with a tool rotation speed of $600 \mathrm{rpm}$ and a dwell time of $15 \mathrm{~s}$ showed a most cathodic reaction was occurring with that spot weld, which would indicate that cathodic protection should be used. The spot weld with a tool rotation of $900 \mathrm{rpm}$ and the as-received material with a dwell time of $15 \mathrm{~s}$ showed more anodic reactions however a disorder is seen on the Tafel plot of $900 \mathrm{rpm}$, which may be an indication as to why the corrosion rate was so high. Furthermore, at $1200 \mathrm{rpm}$ the sample begins to stabilise having a more cathodic reaction. Coincidentally, there is no clear correlation to the increase in the dwell time at a constant tool rotation rate.

\subsection{Mechanical characteristics: tensile shear testing}

The tensile shear strength obtained from the as-received AA6063 material generated from the tensile machine was $110 \mathrm{MPa}$ using the ASTM E8M-13 standard with the testXpert II software. The tensile shear testing for the spot welds produced is seen in Figure 14.

The tensile shear testing data generated for the asreceived AA6063 material was found to be $110 \mathrm{MPa}$. With a reference from the AA6063 datasheet reported in [33], it can be concluded that the material received falls within the standard range for an AA6063 material. Shear testing is conducted to obtain the shear strength of the alloy, which represents the maximum shear stress the material can withstand before it fractures. This test is beneficial in

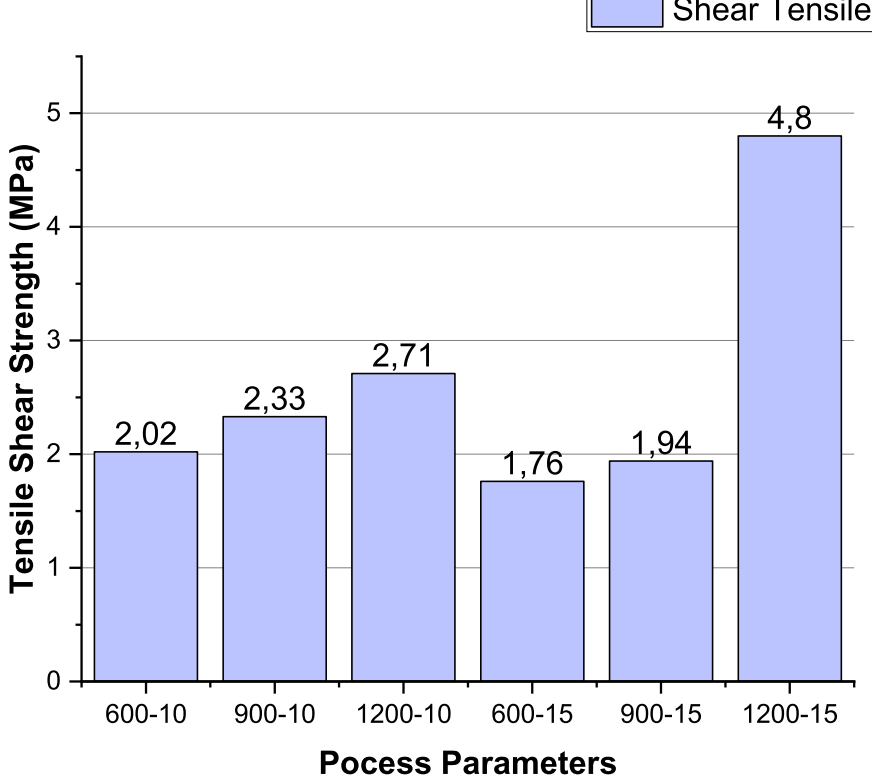

Fig. 14. The tensile shear testing profile for the spot welds produced by FSSW.

knowing which material is best suited for a certain application where an excessive load is applied.

Tensile shear testing for the welds generated is represented by Figure 14. It is shown that the shear strength of the as-received material decreased. This might be because a spot weld was generated with heat and that frictional heat had an influence in the integrity of the material, thus decreasing in the shear strength. One can see that as the tool rotation rate increases at a constant dwell time, the strength increases as well, correlating to the study of Kuleki et al. [12], Liu et al. [13] and Hamzah et al. [17]. The tool rotation rate is a dominant factor in the weld strength and plays a pivotal role in the FSSW process.

Kuleki [12] aimed to establish a relationship between the process parameters and the tensile strength of FSSW AA 5005, Liu et al. [13] examined the microstructural characteristics and mechanical properties of FSSW of AA 2A12-T4 and Hamzah et al. [17] studied how the pin shape and the rotation speed affects the mechanical behaviour and the microstructural analysis of FSSW AA6061. Altogether, these authors concluded that as the rotational tool rate increases, the shear strength increases as well.

Furthermore, it was seen that as the dwell time increases at a fixed rotational tool rate, the tensile shear strength decreased at 600 and $900 \mathrm{rpm}$ however it increased dramatically at $1200 \mathrm{rpm}$. These results were supported by Kuleki et al. [12] yet disproved by Bozkurt et al. [2], Abedin et al. [7]; Bodake and Gujar [15] and Jambhale et al. [32]; in which they stated that as the dwell time increases the tensile shear strength should increase as well then decrease at a certain limit. Kuleki [12] conducted a study on how the tensile shear strength of AA 5005 becomes affected by the process parameters of FSSW, where it was concluded that as the dwell time increased the shear strength decreased. Abedeni et al. [7] studied the effect of tool penetration depth, tool holding time and the pin design on the hook 
geometry on the mechanical properties, Bodake and Gujar [15] wrote a review paper on the optimization of the FSW process parameters, likewise, Jambhale et al. [32] wrote a review paper on how the process parameters and the tool geometries influences on the properties on FSSW; It can be inferred from all the studies that, the dwell time is directly proportional to the weld strength, as the dwell time increases the shear strength increases as well.

The dwell time is the time in which the probe stays rotating at the lower sheet of the overlapping material, with an efficient amount of time, the probe is expected to produce a sound weld which consequently progresses the mechanical properties of the spot weld. This might be a reason as to why the tensile shear strength should increase as the dwell time increases.

\section{Conclusion}

FSSW was successfully performed on overlapping aluminium alloy 6063 pieces with varying tool rotation speed and dwell time. A smooth and clear spot weld was formed as the too rotation rate increased at a constant dwell time, however at $1200 \mathrm{rpm}$ at $15 \mathrm{~s}$ it was found that the keyhole spot weld had fractured. Three major microstructural characteristics were found; the SZ, TMAZ and the HAZ. It was found that more HAZ forms as the tool rotation rate increased at a constant dwell time and more AlMg formulates. The hardest hardness profile was displayed in the SZ zone. At a constant dwelling time, the hardness profile showed an increase towards the centre of the spot weld, then a decrease from the spot weld. The decrease in the hardness profile was due to the different zones present, the TMAZ and the HAZ. The lowest hardness resulted was seen in the HAZ. It was found that the corrosion rate displayed shows that welding at $600 \mathrm{rpm}$ at $10 \mathrm{~s}$ has the lowest corrosion rate of $0.1657 \mathrm{~mm} / \mathrm{yr}$ which means that the material is the most corrosion resistant to ASW.

The corrosion rate displayed further shows that the corrosion rate improves when welding from 900 to $1200 \mathrm{rpm}$ for $15 \mathrm{~s}$ as the corrosion rate decreases from 127.2 to $14.27 \mathrm{~mm} / \mathrm{yr}$. The tensile shear strength presented showed that there are some improvements from 2.02 to $2.71 \mathrm{MPa}$ at $10 \mathrm{~s}$ and from 1.76 to $4.8 \mathrm{MPa}$ at $15 \mathrm{~s}$ ) as the tool rotation rate increases at a constant dwell time.

One may conclude that the tool rotation rate played a more significant role in the testing of the mechanical properties than the dwell time. It can be appreciated that in applications where hardness is significant the ideal spot weld to produce would be welding up to $900 \mathrm{rpm}$ at 10 and/ or $15 \mathrm{~s}$ as finer grains were developed which evidently improved the hardness. In applications where corrosion is significant (especially in the marine industry), the ideal spot weld to produce would be welding at $600 \mathrm{rpm}$ for $15 \mathrm{~s}$ as it was found to have had the lowest corrosion rate which in turn means that the material will be corrosion resistant to seawater. Alternatively, a spot weld from $900 \mathrm{rpm}$ to $1200 \mathrm{rpm}$ at $15 \mathrm{~s}$ would be accepted as the corrosion rate decreases which in turn means that the material will also be corrosion resistant to seawater. In applications where the tensile shear strength is significant the ideal spot weld to produce would be to weld up to $1200 \mathrm{rpm}$ at 10 and/or $15 \mathrm{~s}$ as the tensile shear strength was found to have been improved.

This research is significant to the manufacturing industry, predominantly in the marine, automotive and the aerospace industry where the use of lightweight joint alloys is significant. The success of this study and the understanding of how the process parameters affect the evolving properties of the aluminium alloy would help in establishing which process parameters effectively influence the strength, the mechanical properties and the corrosion behaviour of the aluminium alloy; placing the parts in the corrected fields. This study will improve the operation and profitability of the mentioned industries, as this particular welding technique can produce mass production, leaving little residual stresses onto the weld, producing an excellent surface finish as well as being able to weld both similar and dissimilar materials which will improve the performance of the part to be welded. It will equally generate knowledge and further developments in the welding of aluminium alloys.

The support of the Friction stir welding Laboratory in the department of Mechanical Engineering, Indian Institute of Technology, Kharagpur, Indian and the University of Johannesburg is acknowledged.

\section{References}

1. C. Yeni, O. Ugur, S. Sami, Effect of in penetration depth on the mechanical properties of friction stir spot welded aluminium and copper. Mater. Test. J. Technol. 54 (2012) 233-239

2. Y. Bozkurt, S. Salman, G. Cam, The effect of welding parameters on lap-shear tensile properties of dissimilarfriction stir spot welded AA5754-H22/2024-T3 joints. Sci. Technol. Weld. Joi. 18 (2013) 337-345

3. H. Guler, Influence of the tool geometry and process parameters on the static strength and hardness of friction stir spot welded aluminium alloy Sheets. Mater. Technol. 49 (2014) 457-460

4. I.J. Ibrahim, G.G. Yapici, Applications of a novel friction stir spot welding process on dissimilar aluminium joints. J. Manuf. Proc. 35 (2017) 282-288

5. M.A. Tashkandi, J.A. Al-Jarrah, M. Ibrahim, Spot welding of 6061 aluminium alloy by friction stir spot welding process. Eng. Technol. Appl. Sci. Res 7 (2017) 1629-1632

6. M. Paidar, A. Khodabandeh, M.L. Sarab, M. Taheri, Effect of welding parameters (plunge depths of shoulder, pin geometry and tool rotation speed) on the failure mode and stir zone characteristics of friction stir spot welded aluminium 2024-T3 sheets. J. Mech. Sci. Technol. 29 (2014) 4639-4644

7. O. Abedeni, E. Ranjbarnodeh, P. Marashi, Effect of tool geometry and welding parameters on the microstructure and static strength of the friction stir spot welded DP780 dual phase steel sheets. Mater. Technol. 51 (2017) 687-694

8. M. Awang, V.H. Mucino, Z. Feng, S.A. David, Thermosmechanical modelling of friction stir spot welding (FSSW) process: use of an explicit adaptive meshing scheme. SAE Technical Paper. 2005; 2005-2001-1251 
9. W. Yuang, Friction Stir Spot Welding of Aluminium Alloys (Missouri University of Science and Technology, 2008)

10. S. Siddharth, T. Senthikumar, A study of friction stir spot welding process and its parameters for increasing strength of dissimilar joint. Univ. Zulia 39 (2016) 168-176

11. O.M. Ikumapayi, E.T. Akinlabi, Recent advances in keyhole defect repairs via refilling friction stir spot welding. Mater. Today Proc. 18 (2019) 2201-2208

12. M.K. Kulekci, Effect of process parameters on tensile shear strength of friction stir spot welded Aluminium Alloy (EN AW 5005). Arch. Metall. Mater. 59 (2014) 221-224

13. H. Liu, Y. Zhao, X. Su, L. Yu, J. Hou, Microstructural characteristics and mechanical properties of friction stir spot welded 2A12-T4 aluminium alloy. Adv. Mater. Sci. Eng. 2013 (2013) 1-10

14. R.S. Mishra, Z.Y. Ma, Friction stir welding and processing. Mater. Sci. Eng. 5 (2005) 1-78

15. S.N. Bodake, A.J. Gujar, Review paper on optimization of friction stir welding process parameters. Int. J. Eng. Res. Technol 10 (2017) 611-620

16. R. Kumar, V. Singh, Influence of process parameters on mechanical properties of aluminium alloy AA6063 during friction stir welding. Int. J. Eng. Sci. Res. Technol. (IJESRT) 6 (2016) 150-158

17. M.N. Hamzah, S.H. Bakhy, M.A. Fliayyh, Effect of pin shape and rotational speed on the mechanical behaviour and microstructures of friction stir spot welding of AA6061 aluminium alloy. Al-Nahrain J. Eng. Sci. (NJES) 20 (2017) 129-139

18. G. Buffa, L. Fratini, M. Piacentini, Tool path design in friction stir spot welding of AA6068-T6 aluminium alloys. Key Eng. Mater. 344 (2007) 767-774

19. L. Fratini, A. Barcellona, G. Buffa, D. Palmeri, Friction stir spot welding of AA6082-T6: influence of the most relevant process parameters and comparison with classic mechanical fastening techniques. Proc. IMechE B J. Eng. Manuf. 221 (2007) 1111-1118

20. K. Chen, X. Liu, J. Ni, Effects of process parameters on friction stir spot welding of aluminium alloys to advanced high strength steel. J. Manuf. Sci. Eng. 139 (2017) 081016

21. C. Jonckheere, B. de Meester, C. Cassiers, M. Delhaye, A. Simar, Fracture and mechanical properties of friction stir spot welds in 6063-T6 aluminum alloy. Int. J. Adv. Manuf. Technol. 62 (2012) 569-575

22. K. Chen, X. Liu, J. Ni, Keyhole refilled friction stir spot welding of aluminium alloy to advanced high strength steel. In ASME 2016 International Manufacturing Science and Engineering Conference, 1-11

23. E. Fereiduni, M. Movahedi, A.H. Kokabi, H. Najfi, Effect of dwell time on joint interface microstructure and strength of dissimilar friction stir spot- welded Al-5083 and St-12 alloy sheets. Metall and Mat. Trans. A 48 (2017) 1744-1758

24. S. Ravikumar, V.S. Rao, R.V. Pranesh, Effect of process parameters on mechanical properties of friction stir welded dissimilar materials between AA6061-T651 and AA7075T651 alloys. Int. J. Adv. Mech. Eng. 4 (2014) 101-114
25. M.K. Abbass, S.K. Hussein, A.A. Khudhair, Optimization of mechanical properties of friction stir spot welded joints of dissimilar aluminium alloys (AA2024-T3 and AA5754H114). Res. Article Mech. Metall. 1 (2016) 1-10

26. Z. Li, Z. Xu, L. Zhang, Z. Yan, Friction spot welding of dissimilar 6063/5083 aluminium alloys. Mater. Sci. Technol. 33 (2017) 1626-1634

27. ASTM International Standards, Standard practice for micro etching metals and alloys. designation: E407-07, 2015, 9

28. T. Vuherer, P. Maruschak, I. Samardžić, Behaviour of coarse grain in Heat Affected Zone (HAZ) during cycle loading. Metalurgija 51 (2012) 301-304

29. O. Abedeni, E. Ranjbarnodeh, P. Marashi, Effect of tool geometry and welding parameters on the microstructure and static strength of the friction stir spot welded DP780 dual phase steel sheets. Mater. Technol. 51 (2017) 687-694

30. O.M. Ikumapayi, E.T. Akinlabi, J.D. Majumdar, S.A. Akinlabi, Influence of $17-4 \mathrm{PH}$ stainless steel and $\alpha+\beta$ titanium alloy powders for corrosion susceptibility on friction stir processed AA7075-T651 aluminium matrix composites. J. Bio Tribo Corr. 5 (2019) 1-11

31. S. Siddharth, T. Senthikumar, A Study of Tool Penetration Behaviour in Dissimilar Al5083/C10100 Friction Stir Spot Welds (Elsevier Science Direct, 2017)

32. C. Obayi, R. Tolouei, A. Mostavan, C. Paternoster, S. Turgeon, B.A. Okorie, D. Obikwelu, D. Mantovani, Effect of grain size on mechanical properties and biodegradation behaviour of pure iron for cardiovascular stent application. Bio-Matter. 6 (2016) 1-14

33. S. Jambhale, S. Kumar, S. Kumar, Effect of process parameters and too geometries on properties of friction stir spot review. Univ. J. Eng. Sci. 3 (2015) 7-11

34. ASM Aerospace Specification Metals Inc., 1978. http:// asm.matweb.com/search/SpecificMaterial.asp?bassnum= MA6063T4 (accessed 21 August, 2018)

35. C.U. Atuanya, A.O.A. Ibhadode, I.M. Dagwa, Effects of breadfruit seed hull ash on the microstructures and properties of Al-Si-Fe alloy/breadfruit seed hull ash particulate composites. Elsevier 2 (2012) 142-149

36. R. Kumar, V. Singh, Influence of process parameters on mechanical properties of aluminium alloy AA6063 during friction stir welding. Int. J. Eng. Sci. Res. Technol. (IJESRT) 6 (2016) 150-158

37. T. Bell, How to calculate the rate of metal corrosion, 2018. https://www.thebalance.com/corrosion-rate-calculator2339697 (accessed 30 November, 2018)

38. C.S. Obayi, R. Tolouei, A. Mostvan, C. Patemoster, S. Turgeon, T.B. Okorie, D. Mantovani, Effect of grain sizes on mechanical properties and biodegradation behaviour of pure iron for cardiovascular stent application. Bio Matter. 6 (2014) 1-9

39. O.M. Ikumapayi, E.T. Akinlabi, Efficacy of $\alpha$-b grade titanium alloy powder (Ti-6Al-2Sn-2Zr-2Mo-2Cr-0.25Si) in surface modification and corrosion mitigation in $3.5 \% \mathrm{NaCl}$ on friction stir processed armour grade 7075-T651 aluminium alloys - insight in defence applications. Mater. Res. Exp. 6 (2019) $1-15$

Cite this article as: Delphine Mulaba-Kapinga, Kasongo Didier Nyembwe, Omolayo Michael Ikumapayi, Esther Titilayo Akinlabi, Mechanical, electrochemical and structural characteristics of friction stir spot welds of aluminium alloy 6063, Manufacturing Rev. 7, 25 (2020) 